\title{
Intestinal attraction: CCL25 functions in effector lymphocyte recruitment to the small intestine
}

\author{
Daniel J. Campbell and Eugene C. Butcher \\ Laboratory of Immunology and Vascular Biology, Department of Pathology, Stanford University School of Medicine, Stanford, California, USA, \\ and the Center for Molecular Biology and Medicine, Veterans Affairs Palo Alto Health Care System, Palo Alto, California, USA \\ J. Clin. Invest. 110:1079-1081 (2002). doi:10.1172/JCI200216946.
}

\begin{abstract}
A large surface area and continuous exposure to the outside environment through food intake make the intestines an ideal location for the growth and/or entry of pathogenic microorganisms and parasites. It is therefore not surprising that large numbers of lymphocytes are associated with the intestines within specialized gut-associated lymphoid tissues (GALT) and in the intestines themselves (1). Within the intestines, lymphocytes are localized in two main compartments, the lamina propria (LP) and the intestinal epithelium (IE). Intestinal intraepithelial lymphocytes (IELs) consist mainly of cytotoxic T cells and have been divided into two subsets, type a and type b, based on their mode of antigen recognition (2). Responses of type a IELs are MHC restricted and directed against peptides derived from invading pathogens. Type b IELs display limited $T$ cell receptor (TCR) diversity (both $\alpha \beta$ and $\gamma \delta$ TCRs are utilized), are not restricted by classical MHC molecules, and are thought to be largely autoreactive, recognizing
\end{abstract}

\footnotetext{
Address correspondence to: Daniel J. Campbell, 154B, 3801 Miranda Avenue, Palo Alto, California 94304, USA.

Phone: (650) 493-5000 ext. 63132; Fax: (650) 858-3986;

E-mail: daniel@macampbell.com. Conflict of interest: No conflict of interest has been declared.

Nonstandard abbreviations used: gutassociated lymphoid tissues (GALT); lamina propria (LP); intestinal epithelium (IE); intraepithelial lymphocyte (IEL); T cell receptor (TCR); lamina propria lymphocyte (LPL); antibody-secreting cell (ASC); peripheral lymph node (PLN); Peyer's patches (PP); mesenteric lymph node (MLN); dendritic cell (DC).
}

nonpolymorphic stress-induced proteins displayed by infected or damaged epithelial cells.

Lamina propria lymphocytes (LPLs) and type a IELs are believed to be derived from lymphocytes that recognized antigen in GALT, but the molecules that direct their migration to the intestines are not completely understood (3). In this issue of the JCI, Svensson et al. (4) demonstrate that expression of the chemokine receptor CCR9 is selectively maintained on $\mathrm{CD}^{+} \mathrm{T}$ cells activated in the GALT and that neutralization of the CCR9 ligand CCL25 strongly inhibits the accumulation of these cells in the type a IEL compartment (Figure 1). These results mark the first direct demonstration of a functional role for a chemokine/receptor pair in normal lymphocyte localization to an intestinal effector site.

\section{CCL25 and intestinal lymphocyte recruitment}

Lymphocyte expression of adhesion molecules and chemokine receptors directs their migration from the blood into tissues. Chemokines function at several steps in these homing pathways, mediating firm arrest of lymphocytes rolling along vascular endothelium in postcapillary venules, promoting diapedesis through the endothelium into the underlying tissue parenchyma, and segregating lymphocytes within tissues into functional microenvironments. The best-characterized example of these chemokine functions involves homing into secondary lymphoid tissues, where the chemokines CCL21, CCL19, and CXCL13 (through their lymphocyte receptors CCR7 and CXCR5) mediate the entry of naive lymphocytes and their segregation into distinct $\mathrm{T}$ and $\mathrm{B}$ cell areas (5). Chemokines serve analogous functions in lymphocyte homing to nonlymphoid effector tissues; for example, the chemokines CCL17 and CCL27 together mediate homing of effector lymphocytes into inflamed skin $(6,7)$. Although several chemokines are expressed within the intestines (some quite selectively) and can mediate specific chemotaxis of gut homing lymphocyte populations (8), a direct role for these molecules in intestinal lymphocyte homing has not been established.

Due to its highly restricted expression, CCL25 has attracted considerable attention as a potential intestinespecific homing beacon $(9,10)$. Mice deficient in the CCL25 receptor CCR9 have been generated by two groups, each showing relatively minor (and somewhat conflicting) defects in IELs $(11,12)$. However, in the knockout animals, homeostatic mechanisms may compensate for any decreased lymphocyte homing to the intestinal epithelium. Additionally, some of the defects observed may be due to altered IEL development, since type b IELs are thought to arise in the intestinal cryptopatches, a site of CCL25 expression (13). By neutralizing CCL25 in vivo with a blocking antibody, Svensson et al. (4) directly demonstrate that CCL25 and CCR9 function in recruiting effector lymphocytes to the small intestinal epithelium following their activation in GALT.

The precise role of CCL25 in lymphocyte homing to the IE remains to be determined. In addition to expression by small intestinal IELs, CCR9 is also found on all $\mathrm{CD} 4^{+}$and $\mathrm{CD} 8^{+}$small intestinal LPLs (9), and IgA antibodysecreting cells (ASCs) isolated from 


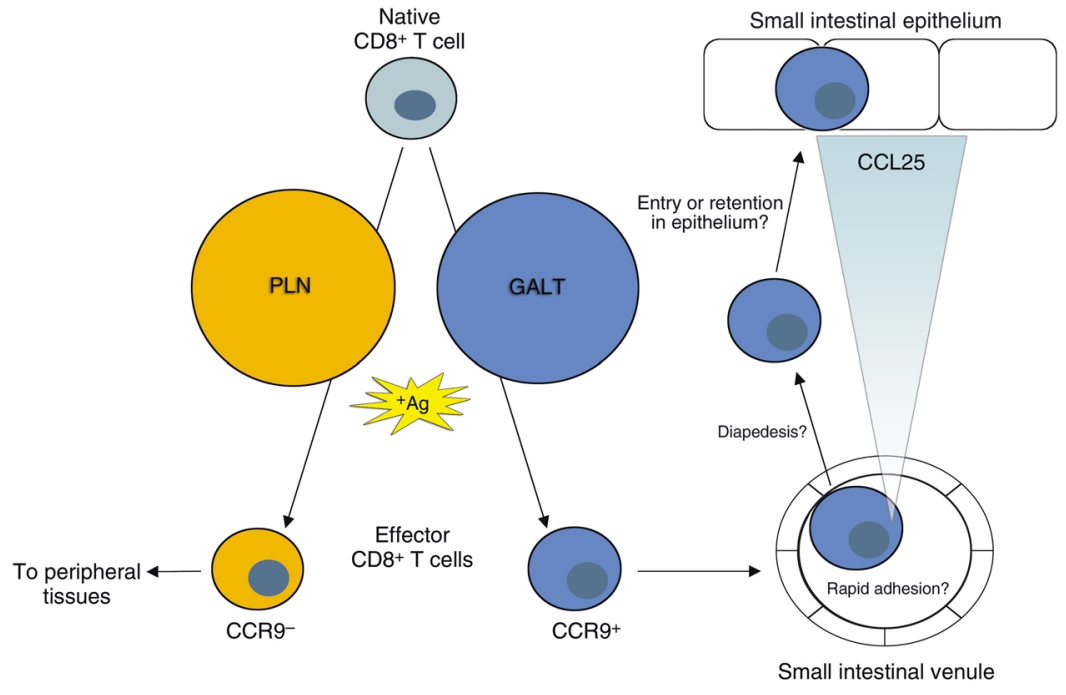

Figure 1

A subset of naive $C D 8^{+} T$ cells recognizing antigen $(\mathrm{Ag})$ in GALT, but not PLNs, selectively retains CCR9 expression and is targeted to the small intestinal epithelium (4). Selective expression of adhesion molecules such as $\alpha_{4} \beta_{7}$ and $\alpha_{E} \beta_{7}$ are also likely to contribute to this tropism (18). Within the small intestine, CCL25 produced by IE mediates homing of effector CD $8^{+} \mathrm{T}$ cells by promoting rapid adhesion, diapedesis, and/or entry of cells into the IE (8).

GALT or the small intestinal LP are CCL25 responsive (14). CCL25 protein is found both within the crypts and lower villi of the intestinal epithelium and on the luminal surface of the vascular endothelium within the human small intestine $(9,10)$. Thus, it seems unlikely that CCL25 and CCR9 function to segregate LPLs and IELs. More likely, CCL25 mediates lymphocyte entry into the small intestine by promoting rapid adhesion in vascular endothelium or transmigration into the LP, where other chemoattractant or adhesive interactions subsequently separate IELs from LPLs. In this regard, it will be interesting to determine the effects of blocking CCL25 on the localization of $\mathrm{CD}^{+} \mathrm{T}$ cells and IgA ASCs to the small intestinal LP. In addition, while many mucosal tissues contain LPLs and IELs, CCL25 is found only in the small intestine, and the chemokines performing analogous functions in other mucosal sites remain to be identified. Of interest is the closely related chemokine CCL28, which is expressed by epithelial cells in several mucosal tissues $(15,16)$.

\section{Lymphocyte activation in the unique GALT environment}

The second major finding of Svensson et al. is that $\mathrm{CD}^{+} \mathrm{T}$ cells activated in GALT (compared with peripheral lymph nodes [PLN]) selectively retain
CCR9 expression (4). This is consistent with classic studies showing that lymphoblasts collected from GALT display intestinal tropism (17) as well as with our own, more recent, work demonstrating that $\mathrm{CD}^{+}{ }^{+} \mathrm{T}$ cells activated in GALT express the intestinal homing integrin $\alpha_{4} \beta_{7}$ and respond to CCL25 (18). In addition, co-expression of CCR9 and IgA suggests that B cells activated in GALT also selectively express this intestinal homing receptor (14). Thus, it appears that GALT are unique among secondary lymphoid tissues in their ability to induce or select for lymphocytes with intestinal homing tropism.

The signals directing lymphocyte expression of CCR9 and $\alpha_{4} \beta_{7}$ in GALT remain poorly understood. CCL25 mRNA has been detected in the follicle-associated epithelium of Peyer's patches (PP) (19), so it is possible that CCR9 expression is regulated through a positive feedback loop. However, CCR9 expression has been observed on $\mathrm{T}$ cells activated in the mesenteric lymph nodes (MLN), which lack this CCL25 $5^{+}$epithelium $(4,18)$. Thus, although positive feedback may help reinforce CCR9 expression on cells activated in PP, a more likely scenario is one in which dendritic cells (DCs) presenting antigens in GALT express an intestinespecific complement of costimulato- ry molecules and cytokines. Upon $\mathrm{T}$ cell activation, these DCs then direct (or select for) $\mathrm{T}$ cell expression of appropriate intestinal adhesion and chemokine receptors. During B cell activation and differentiation in germinal centers, $\mathrm{CD} 4^{+} \mathrm{T}$ cells can then pass this information along to antigen-specific B cells, along with instructions to switch to the mucosal isotype IgA. Consistent with this model, induction of mucosal versus peripheral $\mathrm{CD}^{+}$cytotoxic effector $\mathrm{T}$ cells depends on different sets of costimulatory interactions (20).

\section{Concluding remarks}

Lymphocyte homing to the intestines is critical for defense against enteric pathogens. However, these same processes likely contribute to the pathology of intestinal autoimmune and inflammatory diseases. Thus, chemokines such as CCL25 that participate in recruitment of intestinal lymphocytes make attractive targets for development of tissue-specific anti-inflammatory drugs. In addition, the development of gut-tropic lymphocytes in GALT represents a poorly characterized pathway of lymphocyte differentiation, with implications for production of effective mucosal and systemic vaccines. Further study of these processes will certainly lead to new insights into lymphocyte biology and the complex interplay between the immune system and mucosal tissues.

\footnotetext{
1. Nagler-Anderson, C. 2001. Man the barrier! Strategic defences in the intestinal mucosa. Nat. Rev. Immunol. 1:59-67.

2. Hayday, A., Theodoridis, E., Ramsburg, E., and Shires, J. 2001. Intraepithelial lymphocytes: exploring the Third Way in immunology. Nat Immunol. 2:997-1003.

3. Butcher, E.C., Williams, M., Youngman, K., Rott, L., and Briskin, M. 1999. Lymphocyte trafficking and regional immunity. Adv. Immunol. 72:209-253.

4. Svensson, M., et al. 2002. CCL25 mediates the localization of recently activated $\mathrm{CD} 8 \alpha \beta^{+}$lymphocytes to the small-intestinal mucosa. J. Clin. Invest. 110:1113-1121. doi:10.1172/JCI200215988.

5. Muller, G., Hopken, U.E., Stein, H., and Lipp, M. 2002. Systemic immunoregulatory and pathogenic functions of homeostatic chemokine receptors. J. Leukoc. Biol. 72:1-8.

6. Homey, B., et al. 2002. CCL27-CCR10 interactions regulate $\mathrm{T}$ cell-mediated skin inflammation. Nat. Med. 8:157-165.

7. Reiss, Y., Proudfoot, A.E., Power, C.A., Campbell, J.J., and Butcher, E.C. 2001. CC chemokine receptor (CCR) 4 and the CCR10 ligand cutaneous T cell-attracting chemokine (CTACK) in lymphocyte trafficking to inflamed skin. J. Exp. Med. 194:1541-1547.

8. Kunkel, E.J., and Butcher, E.C. 2002. Chemokines
} 
and the tissue-specific migration of lymphocytes. Immunity. 16:1-4.

9. Kunkel, E.J., et al. 2000. Lymphocyte CC chemokine receptor 9 and epithelial thymusexpressed chemokine (TECK) expression distinguish the small intestinal immune compartment: Epithelial expression of tissue-specific chemokines as an organizing principle in regional immunity. J. Exp. Med. 192:761-768.

10. Papadakis, K.A., et al. 2000. The role of thymusexpressed chemokine and its receptor CCR9 on lymphocytes in the regional specialization of the mucosal immune system. J. Immunol. 165:5069-5076

11. Uehara, S., Grinberg, A., Farber, J.M., and Love, P.E. 2002. A role for CCR9 in T lymphocyte development and migration. J. Immunol. 168:2811-2819.

12. Wurbel, M.A., et al. 2001. Mice lacking the CCR9
CC-chemokine receptor show a mild impairment of early T- and B-cell development and a reduction in T-cell receptor $\gamma \delta(+)$ gut intraepithelial lymphocytes. Blood. 98:2626-2632.

13. Onai, N., et al. 2002. Pivotal role of CCL25 (TECK)-CCR9 in the formation of gut cryptopatches and consequent appearance of intestinal intraepithelial T lymphocytes. Int. Immunol. 14:687-694.

14. Bowman, E.P., et al. 2002. The intestinal chemokine thymus-expressed chemokine (CCL25) attracts IgA antibody-secreting cells. J. Exp. Med. 195:269-275.

15. Pan, J., et al. 2000. A novel chemokine ligand for CCR 10 and CCR 3 expressed by epithelial cells in mucosal tissues. J. Immunol. 165:2943-2949.

16. Wang, W., et al. 2000. Identification of a nove chemokine (CCL28), which binds CCR10 (GPR2). J. Biol. Chem. 275:22313-22323.
17. Griscelli, C., Vassalli, P., and McCluskey, R.T 1969. The distribution of large dividing lymph node cells in syngeneic recipient rats after intravenous injection. J. Exp. Med. 130:1427-1451.

18. Campbell, D.J., and Butcher, E.C. 2002. Rapid acquisition of tissue-specific homing phenotypes by $\mathrm{CD} 4(+) \mathrm{T}$ cells activated in cutaneous or mucosal lymphoid tissues. J. Exp. Med. 195:135-141.

19. Wurbel, M.A., et al. 2000. The chemokine TECK is expressed by thymic and intestinal epithelial cells and attracts double- and single-positive thymocytes expressing the TECK receptor CCR9. Eur. J. Immunol. 30:262-271.

20. Kim, S.K., et al. 1998. Generation of mucosal cytotoxic $\mathrm{T}$ cells against soluble protein by tissue-specific environmental and costimulatory signals. Proc. Natl. Acad. Sci. USA. 95:10814-10819. 